\title{
LA-8292-MS
}

DR. 1037

Informal Report
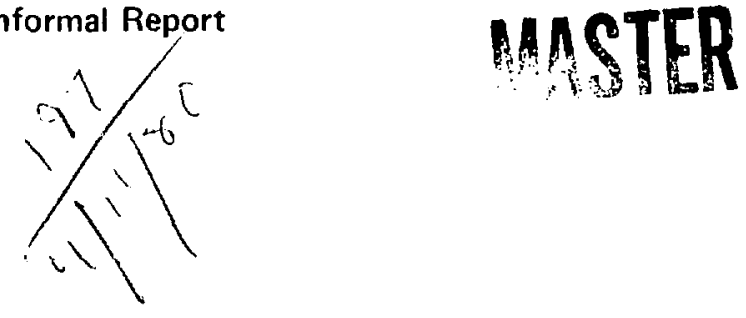

The Effect of Model Choice and Sample Size on Statistical Tolerance Limits
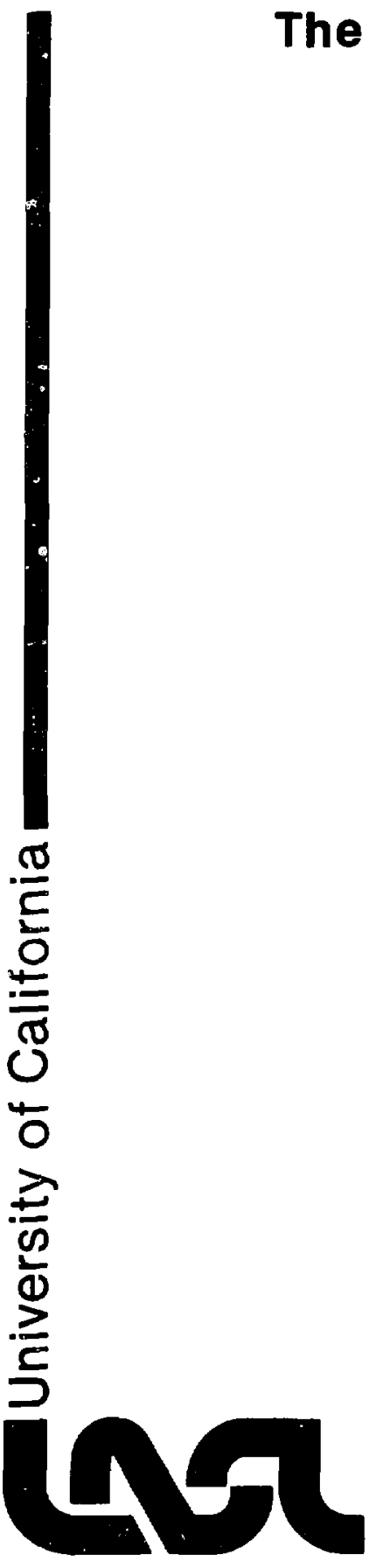
LA-8292-MS

Informal Report

UC-79p (Base Technolog Issued: March 1980

\section{The Effect of Model Choice and Sample Size on Statistical Tolerance Limits}

Benjamin S. Duran*

Katherine Campbelt

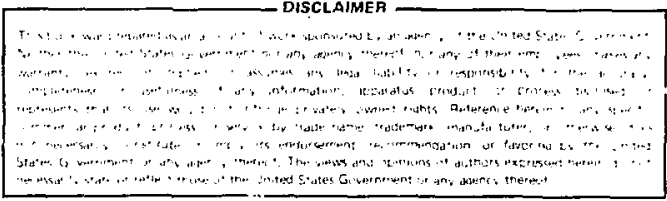

*Visiting Staff Member, Department of Mathematics, Texas Tech University, Lubbock, TX 79409.

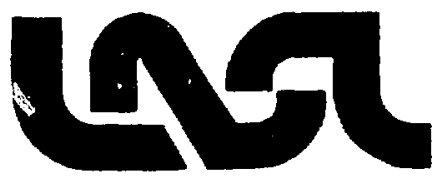


THE EFFECT OF MODEL CHOICE AND SAMPLE SIZE

ON STATISTICAL TOLERANCE LIMITS

by

Benjamin S. Duran and Katherine Campbell

\begin{abstract}
Statistical tolerance 1 imits are estimates of large (or small) quantiles of a distribution, quantities which are very sensitive to the shape of the tail of the distribution. The exact nature of this tail behavior cannot be ascertained from small samples, so statistical tolerance limits are frequently computed using a statistical model chosen on the bas is of theoretical considerations or prior experience with similar populations. This report illustrates the effects of such choices on the computations.
\end{abstract}

\title{
I. INTRODUCTION
}

In life testing and in reliability studies an estimate of a lower bound, such that a large fraction of the life lengths of the items under study $c$ an be expected to fall above this bound, is frequently required. In other contexts an upper bound for the bulk of the population may te of more interest. Estimates of these bounds based on data are called lower or upper tolerance limits, respectively. (Two-sided tolerance limits, which estimate a finite interval containing a large fraction of the population, are aiso used, although they will not be discussed here.)

Usually the fraction of the population to be bounded is large (at least $90 \%)$, and so the bounds are rather sensitive to the "tail" behavior of the underlying distribution. Unfortunately, the data are also frequentiy sparse, and under these circumstances it is impossible to reject one type of probabi1ity model in favor of another, even when the tail behaviors of the two candidates are radically different. Therefore, the analyst is forced to assume a model based on theoretical considerations, previous experience, or simple 
guesswork. The purpose of this paper is to develop the reader's intuition about the effect of such choices on the computed tolerance limits. Most of the theoretical discussion and derivations are placed in App. A and B. The text develops tolerance limits for two sets of data under several different types of assumptions.

The following paragraphs provide alternative definitions of tolerance limits, from both the classical and the Bayesian points of view.

Definition 1.1. A (classical) lower $(P, Y)$ tolerance limit is a random variable, a function $L(\underset{\sim}{X})$ of observations $\underset{\sim}{X}=\left(x_{1}, x_{2}, \ldots, x_{n}\right)$ from an unknown distribution $F$, such that

$$
\operatorname{Pr}\left\{\int_{L(\underset{\sim}{X})}^{\infty} d F(x) \geq P\right\}=\operatorname{Pr}\{1-F(\underset{\sim}{L(X)}) \geq P\}=\gamma .
$$

Similarly, an upper $(P, \gamma)$ tolerance limit is a function $U(\underset{\sim}{X})$ such that

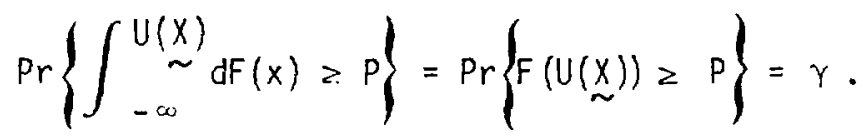

Let $\xi_{p}$ denote the $p^{\text {th }}$ quantile of the distribution $F$. That is, $\xi_{p}$ and $\xi_{1-p}$ satisfy

$$
P \leq \operatorname{Pr}\left\{x \leq \xi_{p}\right\}=\int_{-\infty}^{\xi_{p}} \mathrm{dF}(x)
$$

and

$$
P \leq \operatorname{Pr}\left\{X \geq \xi_{1-P}\right\}=\int_{\xi_{1-P}}^{\infty} \mathrm{dF}(x)
$$

(If $F$ is a continuous distribution, the first inequalities become equalities.) Comparing these equations with those in Def. 1.1 shows that tolerance limits may alternatively be defined as confidence limits for the corresponding quantiles. 
Definition 1.2. A lower $(P, \gamma)$ tolerance limit is a lower $100 \%$ confidence bound for $\xi_{1-P}$, that is,

$$
\operatorname{Pr}\left\{L(\underset{\sim}{X}) \leq \xi_{1-P}\right\}=\gamma \text {. }
$$

Similarly, an upper $(P, Y)$ tolerance limit is an upper $100 \%$ confidence bound for $\xi_{p}$,

$$
\operatorname{Pr}\left\{U(\underset{\sim}{X}) \geq \xi_{p}\right\}=r
$$

This equivalence will prove very useful.

In the classical context of the above definitions, it is not correct to say that the probability is $\gamma$ that the interval $[L(\underset{\sim}{x}), \infty)$ includes $\xi_{1-P}$ (or includes a fraction $P$ of the distribution $F$ ) after the observations $\underset{\sim}{X}=\underset{\sim}{x}$ are available and the function $L(\underset{\sim}{X})$ has been evaluated to get $L(\underset{\sim}{x})$. The above definitions are statements about the sampling distribution of the random variable $L(\underset{\sim}{X})$. Any single observation of $L(X)$, that is, $L(\underset{\sim}{X})$, may or may not be less than or equal to $\xi_{1-p}$. But before the observations are made, the "odds" are $\gamma$ to $1-\gamma$ in favor of obtaining a sample $\underset{\sim}{x}$ such that $L(\underset{\sim}{x})$ is less than or equal to $\xi_{1-P}$, and thus a lower bound for a fraction $P$ or more of the whole population.

Superficially, the definitions of Bayesian tolerance limits, which will also be considered, look much like Def. 1.1 and 1.2. However, the probabilities denoted by $\mathrm{Pr}$ in the defining equations below are to be interpreted as Bayesian prior or posterior probabilities. Thus, Eq. (1.5) is a statement about the posterior distribution of $\xi_{1-P}$ (which still denotes the $(1-P)^{\text {th }}$ quantile of the sampling distribution $F$, but which is a random variable in the Bayesian framework), whereas Eq. (1.3) was a statement about the sampling distribution of the random variable $L(\underset{\sim}{X})$.

Definition 1.3. A (posterior Bayesian) lower $(P, \gamma)$ tolerance limit is a function $L(X)$ such that

$$
\operatorname{Pr}\left\{\xi_{1-P} \geq L(\underset{\sim}{)}) \mid \underset{\sim}{x}=\underset{x}{x}\right\}=r .
$$

Similarly, an upper $(P, \gamma)$ tolerance limit is a function $U(\underset{\sim}{X})$ such that 


$$
\operatorname{Pr}\left\{\xi_{p} \leq U(X) \mid \not X=\not X\right\}=\gamma .
$$

In the Bayesian context it is also possible to compute tolerance limits based solely on the assumed prior distribution (that is, before observing data.) Definition 1.4. A (prior Bayesian) lower $(P, Y)$ tolerance limit is the $(1-\gamma)^{\overline{t h}}$ quantile of the prior distribution of $\xi_{1-p}$,

$$
\operatorname{Pr}\left\{\xi_{1-P} \geq L\right\}=r
$$

Similarly, an upper $(P, \gamma)$ tolerance limit $U$ is the $\gamma^{\text {th }}$ quantile of the prior distribution of $\xi_{p}$,

$$
\operatorname{Pr}\left\{\xi_{p} \leq u\right\}=r
$$

In the Bayesian context, it is correct to say that the (posterior) probability is $\gamma$ that $\left[L(\underset{\sim}{x}), \infty\right.$ ) includes $\xi_{1-P}$ (or includes a fraction of at least $P$ of the population).

It will be seen that the functions $L$ and $U$ of Def. 1.3 are functions of the posterior distribution of the parameters of the sampling distribution $F$. In particular, the Bayesian approach assumes that $F$, al though not completely known, belongs to a parametric family $\left\{F_{\theta}\right\}_{\theta \varepsilon \Theta}$ of distributions indexed by a set of $k$-vectors $\theta=\left\{\theta=\left(\theta_{1}, \ldots, \theta_{k}\right)\right\}$. The $p^{\text {th }}$ quantile of $F_{\theta}$ is then a function of $\underset{\sim}{\theta}$.

The classical approach may also assume such a parametric model. We will consider first, however, a completely nonparametric approach which makes no assumptions about the distribution $F$ of the observations, requiring only that the observations be independent and identically distributed. The results itainable in this case depend strongly on sample size, and are not very satisfactory for small samples. Selection of a parametric model, however, presupposes a great deal about the tail behavior of $F$ (as we shall attempt to illustrate in Sec. III), and therefore in Sec. IV we consider an assumption which is weaker than the specification of a parametric family. In Section $V$ we compare the Bayesian treatment of parametric models to the classical results of Sec. III. Finally, in Sec. VI we make some further remarks concerning sample size for parametric models. 
The examples, discussion of which forms the bulk of the text, are based on two sets of data given in App. C. The first set consists of thiiteen failure rate estimates for pumps from several published sources, ranging over three orders of magnitude. We will suppose, for the sake of argument, that these thirteen numbers form a sample from an underlying distribution $F$, and we will be interested in upper tolerance limits for $F$. The second set of data is a severely censored collection of failure times for 268 diaphragm valves in the chemical and volume control systems of two similar nuclear power plants. Again, we will assume that these failure times are independently and identically distributed according to a distribution $F$, and we will seek lower tolerance limits for this distribution.

\section{NONPARAMETRIC TOLERANCE INTERVALS}

It is instructive to begin our investigation by seeing what conclusions can be drawn while making no assumptions at all about the underlying distribution generating the observations. The discussion below assumes only that the observations are independent and identically distributed.

A nonparametric lower $(P, \gamma)$ tolerance limit is provided by any order statistic $L \underset{\sim}{(X)}=X_{(k)}$ such that

$$
\operatorname{Pr}\left\{X(k) \leq \xi_{1-P}\right\} \geq \gamma
$$

(See Ref. 1. The order statistics of the sample $\underset{\sim}{X}$ are obtained by rearranging the observations in increasing order, so the $k^{\text {th }}$ order statistic, denoted by $x_{(k)}$, is the $k^{\text {th }}$ observation in this sequence.) Notice that it is not possible, in general, to obtain equality in (2.1).

The left-hand side of Eq. (2.1) can be evaluated as follows. By definition of $\xi_{1-P}$,

$$
\operatorname{Pr}\left\{x<\xi_{1-P}\right\} \leq 1-P,
$$

while

$$
\operatorname{Pr}\left\{X \leq \xi_{1-P}\right\} \geq 1-P .
$$


(Equality holds in both cases if the distribution is continuous, but the assumption of continuity is unnecessary.) Since $x_{(k)} \leq \xi_{1-p}$ if and only if at least $k$ of the $n$ observations are less than or equal to $\xi_{1-p}$, and each observation has probability at least 1 - $P$ of being less than or equal to $\xi_{1-p}$,

$$
\begin{aligned}
\operatorname{Pr}\left\{X_{(k)}\right. & \left.=\xi_{1-p}\right\} \\
& =\sum_{j=k}^{n} \operatorname{Pr}\left\{\text { exactiy } j \text { observations are } s \xi_{1-p}\right\} \\
& \geq \sum_{j=k}^{n}\left(\begin{array}{l}
n \\
j
\end{array}\right)(1-P)^{j} p^{n-j} \\
& =1-\sum_{j=0}^{k-1}\left(\begin{array}{l}
n \\
j
\end{array}\right)(1-P)^{j} p^{n-j} .
\end{aligned}
$$

Provided, then, that

$$
\sum_{j=0}^{k-1}\left(\begin{array}{l}
n \\
j
\end{array}\right)(1-P)^{j} p^{n-j} \leq 1-\gamma,
$$

$x_{(k)}$ is a nonparametric lower $(P, \gamma)$ tolerance limit.

Unfortunately, when $n$ is small it is impossible to obtain a lower $(P, \gamma)$ tolerance limit for some large values of $P$ and any reasonable level of confidence $\gamma$, because even when $k=1$, Eq. (2.2) becomes

$$
p^{n} \leq 1-\gamma,
$$

which cannot be satisfied for any $P>(1-\gamma)^{1 / n}$. When $\gamma \geq 0.9$ and $n$ is small, $(1-Y)^{1 / n}$ is much less than 1 . In fact, Eq. (2.3) can be solved for 
$\mathrm{n}$ to obtain minimum sample sizes for the existence of nonparametric lower $(P, \gamma)$ tolerance limits (see Table XV).

Similar results hold for nonparametric upper tolerance bounds. $U(X)=X_{(k)}$ is a nonparametric upper $(P, Y)$ tolerance bound for the distribution if

$$
\operatorname{Pr}\left\{x(k) \geq \xi_{p}\right\} \geq \gamma
$$

Since $\operatorname{Pr}\left\{x \geq \xi_{p}\right\} \geq 1-p$ for each observation, the left side of Eq. (2.4) is bounded below by

$$
\sum_{j=n-k+1}^{n}\left(\begin{array}{l}
n \\
j
\end{array}\right)(1-p)^{j} p^{n-j}=1-\sum_{j=0}^{n-k}\left(\begin{array}{l}
n \\
j
\end{array}\right)(1-P)^{j} p^{n-j},
$$

so $X_{(k)}$ is a nonparametric upper $(P, Y)$ tolerance limit provided that

$$
\sum_{j=0}^{n-k}\left(\begin{array}{l}
n \\
j
\end{array}\right)(1-p)^{j} p^{n-j} \leq 1-\gamma .
$$

Aga in the inequality (2.5) cannot be satisfied, even for $k=n$, unless Eq. (2.3) holds.

Example 1. Data Set I contains only thirteen observations. If $\gamma \geq 0.90$ nonparametric upper $(P, \gamma)$ tolerance limits are unobtainable for

$$
P>(0.1)^{1 / 13}=0.838 \text {. }
$$

As a result, the only entries in Table I are for $P=0.75$, where $X_{(13)}$ $=1.4 \times 10^{-4} \mathrm{f} / \mathrm{h}$ can be used for $\gamma<0.976$.

Example 2. Data Set II, for which $n=268$, is large, but only the first ten order statistics are known because of censoring. These do provide reasonable nonparametric lower $(P, \gamma)$ tolerance bounds for a small range of $P$. The first percentile $(P=0.99)$ cannot be bounded at a level of confidence greater than

$$
\gamma=1-(.99)^{268}=.932
$$


TABLE I

NONPARAMETRIC UPPER TOLERANCE LIMITS FOR DATA SET I

\begin{tabular}{c|ccc}
$\ddots Y$ & & & \\
$\mathrm{P}$ & 0.90 & 0.95 & 0.99 \\
\hline 0.75 & $1.4 \times 10^{-4}$ & $1.4 \times 10^{-4}$ & $-\mathrm{a}$ \\
0.90 & - & - & - \\
0.95 & - & - & -
\end{tabular}

andicates that the given tolerance limit cannot be computed because the sample is too small.

A lower $(0.99,0.90)$ tolerance bound is provided by $X_{(i)} \cdot(0.95, \gamma)$ tolerance bounds are provided by $x_{(9)}, X_{(8)}$, and $X_{(6)}$ at increasing levels of $Y$. Finally, since

$$
\sum_{j=0}^{9}\left(\begin{array}{c}
268 \\
j
\end{array}\right)(0.90)^{268-j}(0.10)^{j}<0.0001
$$

order statistics greater than $X_{(10)}$ should be used for the $(0.90, Y)$ tolerance limits, but these are unavailable, so $X_{(10)}$ is used in Table II.

It is clear that the inferences that are possible using these minimal assumptions (that is, assuming only independent and identically distributed observations) are very limited. To make further progress it will be necessary to introduce additional assumptions, perhaps in the form of classical or Bayesian parametric models. These assumptions can never be tested adequately with small data sets like those considered here. Therefore, improved tolerance limits are possible only at the expense of relying on theoretical arguments, past experience, or guesswork. It is not our intention to argue that these are invalid, but rather to show how the resulting statistics depend on these choices, especially when the samples are small.

\section{CLASSICAL PARAMETRIC TOLERANCE LIMITS}

In this section we consider some of the commonly used probability models for non-negative random variables such as lifetimes. This is not intended to be an exhaustive survey; only some of the more tractable models are included. 8 
TABLE II

NONPARAMETRIC LOWER TOLERANCE LIMITS FOR DATA SET II .

\begin{tabular}{c|ccc}
$\because \gamma$ & & & \\
$\underline{p}$ & 0.90 & 0.95 & 0.99 \\
\hline 0.90 & $1.68 \times 10^{4}$ & $1.68 \times 10^{4}$ & $1.68 \times 10^{4}$ \\
0.95 & $1.61 \times 10^{4}$ & $1.31 \times 10^{4}$ & $0.82 \times 10^{4}$ \\
0.99 & $0.28 \times 10^{4}$ & $-a$ & -
\end{tabular}

andicates that the given tolerance limit cannot be computed because the sample is too small.

The simplest of all non-negative probability models is the exponential distribution, with a single positive parameter $\theta$ and probability density function

$$
f_{\theta}(x)=\theta e^{-\theta x}, x>0
$$

The cumulative distribution function is

$$
\begin{aligned}
F_{\theta}(x) & =\operatorname{Pr}\{x \leq x\} \\
& =\int_{0}^{x} \theta e^{-\theta x} d x \\
& =1-e^{-\theta x} \text { for } x>0 .
\end{aligned}
$$

This model describes the occurrences of events which take place "randomly" in time. In particular, if the lifetime $X$ of an item has an exponential distribution, then the conditional distribution of its "future" life $x-x_{0}$, given that it has survived to time $x_{0}$, is also exponential with the same parameter $\theta$ as $X$ itself. Such an item exhibits neither "burn-in" (failure rate decreasing with time) nor "wear-out" (increasing failure rate.) It is a reasonable and widely used model for certain types of components. (Discussions 
of situations where this and other probability models are appropriate are found in Refs. 2 and 3.)

An exponential distribution is completely characterized by its single paranieter $\theta$; if $\theta$ were known, all quantiles of the distribution would be known as well. Confidence bounds on $\theta$ can be translated directly into confidence bounds on the quantiles (that is, into tolerance limits), as shown in App. A.

Consider a set of data subject to Type II censoring, that is, observation ends at the time of the $r^{\text {th }}$ failure for some $r, 1 \leq r \leq n$. The maximum likelihood estimator for $\theta$ is then

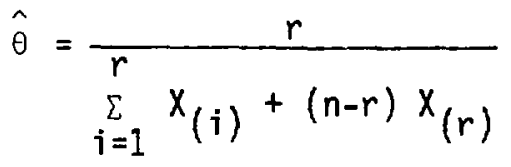

and $2 r \theta / \hat{\theta}$ has a chi-square distribution with $2 r$ degrees of freedom. From this result lower and upper $(P, Y)$ tolerance limits for the distribution are easily derived (see Eq̣s. (A.6) and (A.7) in App. A).

Example 3. Data Set $I$ has no censoring, so $n=r=13$ and $\hat{\theta}=$ $6.56 \times 10^{4}$. Upper $(P, \gamma)$ tolerance limits based on the exponential distribution are given in Table III. The $(0.75,0.95)$ limit is one fourth of the nonparametric limit of Table I, and even the $(0.95,0.99)$ limit is smaller than $X_{(13)}$.

Example 4. In Data Set II, $n=268, r=10$ and $\hat{\theta}=2.255 \times 10^{-6}$. Lower tolerance limits based on the exponential model are shown in Table IV. Where the appropriate order statistics were available in the nonparametric

TABLE III

UPPER TOLERANCE LIMITS FOR DATA SET I BASED ON THE EXPONENTIAL MODEL

\begin{tabular}{c|ccc}
$\ddots \gamma$ & 0.90 & 0.95 & 0.99 \\
\hline 0.75 & $3.18 \times 10^{-5}$ & $3.57 \times 10^{-5}$ & $4.50 \times 10^{-5}$ \\
0.90 & $5.28 \times 10^{-5}$ & $5.93 \times 10^{-5}$ & $7.48 \times 10^{-5}$ \\
0.95 & $6.86 \times 10^{-5}$ & $7.72 \times 10^{-5}$ & $9.73 \times 10^{-5}$
\end{tabular}


case (that is, for $P=0.95$ at all three levels of confidence and for $P=0.99$ and $\gamma=0.90$ ), the entries in Table IV are fairly close to those in Table II.

If we are unwilling to assume that the failure rate is constant, we may wish to consider one of two generalizations of the exponential distribution, the gamma distribution or the Weibull distribution. Both of these distributions have two parameters, a scale parameter $\theta$ and a shape parameter $\alpha$. When $\alpha=1$, both reduce to the exponential distribution with parameter $\theta$, and when $\alpha>1$ both have increasing failure rates. If $\alpha$ is known (or assumed), both of these families are reduced to one-parameter families of distributions, and tolerance limits can be estimated by the methods outlined in App. A.

First, we consider the case where the observations $x_{1}, \ldots, x_{n}$ are uncensored and come from a gamma distribution with known shape parameter $\alpha$. The maximum likelihood estimator for the scale parameter $\theta$ is given in this case by

$$
\hat{\theta}=\frac{\alpha}{\bar{X}}
$$

and $n \alpha \theta \hat{\theta}$ has a gamma distribution with shape parameter $n \alpha$ and scale parameter 1. When $\alpha=k / 2$ for some integer $k$, it is possible to use the more commonly tabulated chi-square distrihution for computation (see App. A), and so we proceed under this assumption, although there is no theoretical difficulty with other values of $\alpha$. In this case lower and upper tolerance limits are given by Eqs. (A.11) and (A.12).

TABLE IV

LOWER TOLERANCE LIMITS FOR DATA SET II BASED ON THE EXPONENTIAL MODEL

\begin{tabular}{c|ccc}
$Y \gamma$ & 0.90 & 0.95 & 0.99 \\
\hline 0.90 & $3.29 \times 10^{4}$ & $2.97 \times 10^{4}$ & $2.49 \times 10^{4}$ \\
0.95 & $1.60 \times 10^{4}$ & $1.45 \times 10^{4}$ & $1.21 \times 10^{4}$ \\
0.99 & $0.31 \times 10^{4}$ & $0.28 \times 10^{4}$ & $0.24 \times 10^{4}$
\end{tabular}


Example 5. Upper tolerance bounds for Data Set $I$, based on gamma distributions with $\alpha=0.5$ and $\alpha=2.0$, are given in Table $v$. The corresponding estimates of $\theta$ are $\hat{\theta}_{0.5}=3.28 \times 10^{4}$ and $\theta_{2}=13.12 \times$ $10^{4}$. Table $V$ should be compared with Table III for the exponential model (that is, $\alpha=1$ ). As $\alpha$ increases the shape of the presumed distribution becomes more peaked and lighter tailed, so that the large quantiles (and their estimates) are pulled down towards the center of the distribution. Thus, the upper $(0.90,0.95)$ tolerance limit goes from $9.10 \times 10^{-5}$ for $\alpha=0.5$ to 5.93 $\times 10^{-5}$ for $\alpha=1$ to $4.23 \times 10^{-5}$ for $\alpha=2$.

When the observations come from a Weibull distribution with known shape parameter $\alpha$, then $x^{\alpha}$ is exponentially distributed with parameter $\theta^{\alpha}$, and lower and upper tolerance limits for the distribution follow imediately from those for the exponential. In particular, the maximum likelihood estimate of $\theta$ is

$$
\hat{\theta}=\left(\frac{r}{\sum_{i=1}^{r} x_{(i)}^{\alpha}+(n-r) x^{\alpha}(r)}\right)^{1 / \alpha} .
$$

Tolerance limits for the Weibull distribution are given in Eqs. (A.13) and (A.14), where $\hat{\beta}=\hat{\theta}^{\alpha}$.

TABLE $V$

UPPER TOLERANCE LIOHITS FOR DATA SET I BASED ON THE GAMMA MODEL WITH SHAPE PARAMETER $\alpha=0.5(\alpha=2$.

\begin{tabular}{l|ccc} 
& & & \\
0.75 & 0.90 & 0.95 & 0.99 \\
\hline \multirow{2}{*}{0.90} & $3.72 \times 10^{-5}$ & $4.45 \times 10^{-5}$ & $6.38 \times 10^{-5}$ \\
& $\left(2.71 \times 10^{-5}\right)$ & $\left(2.93 \times 10^{-5}\right)$ & $\left(3.41 \times 10^{-5}\right)$ \\
& $7.61 \times 10^{-5}$ & $9.10 \times 10^{-5}$ & $13.05 \times 10^{-5}$ \\
& $\left(3.91 \times 10^{-5}\right)$ & $\left(4.23 \times 10^{-5}\right)$ & $\left(4.93 \times 10^{-5}\right)$ \\
& $10.81 \times 10^{-5}$ & $12.92 \times 10^{-5}$ & $18.53 \times 10^{-5}$ \\
$\left(4.77 \times 10^{-5}\right)$ & $\left(5.16 \times 10^{-5}\right)$ & $\left(6.02 \times 10^{-5}\right)$
\end{tabular}


Example 6. Upper tolerance limits for Data Set 1 , based on the Weibull mode1, are given in Table VI for two choices of $\alpha, \alpha=0.5$ and $\alpha=2$. The corresponding estimates of $\theta$ are $\hat{\theta}_{0.5}=13.09 \times 10^{4}$ and $\hat{\theta}_{2}=2.55 \times 10^{4}$. Here if we compare the $(0.90,0.95)$ tolerance limits for the three values of (including $\alpha=1$ from Table III) we may make the unexpected observation that this bound is not a monotonic function of $\alpha$, despite the fact that (as for the garma distribution) larger values of $\alpha$ correspond to more peaked, lightertailed distributions and the larger quantiles should be pulled towards the center of the distribution. In fact, we observe

$$
U(0.90,0.95)=\left\{\begin{array}{l}
11.57 \times 10^{-5} \text { for } \alpha=0.5 \\
5.93 \times 10^{-5} \text { for } \alpha=1.0 \\
7.31 \times 10^{-5} \text { for } \alpha=2.0 .
\end{array}\right.
$$

The problem appears to be that our estimate of the center of the distribution (related to the inverse of the estimate of $\theta$ ) is increasing faster than the increase in $\alpha$ can pull in the tail of the distribution for $\alpha>1$. This is because the estimate $\hat{\theta}$ depends on the sum of the $x_{i}^{\alpha}{ }^{\alpha} s$, and when $\alpha$ is large one anomalously large observation (in this case, $x_{(13)}=1.4 \times 10^{-4}$ ) can dominate this sum. Our estimation procedure in this case is not very "robust", being stronaly influenced by a single extreme value.

\section{TABLE VI}

UPPER TOL.ERANCE BOUNDS FOR DATA SET I BASED ON THE WEIBULL MODEL WITH SHAPE PARAMETER $\alpha=0.5(\alpha=2$.)

\begin{tabular}{c|ccc}
$\ddots \gamma$ & 0.90 & 0.95 & 0.99 \\
\hline 0.75 & $3.32 \times 10^{-5}$ & $4.20 \times 10^{-5}$ & $6.67 \times 10^{-5}$ \\
$\left(5.67 \times 10^{-5}\right)$ & $\left(5.01 \times 10^{-5}\right)$ & $\left(6.75 \times 10^{-5}\right)$ \\
0.90 & $\begin{array}{ccc}1 \\
9.16 \times 10^{-5}\end{array}$ & $11.57 \times 10^{-5}$ & $18.40 \times 10^{-5}$ \\
$\left(7.31 \times 10^{-5}\right)$ & $\left(7.75 \times 10^{-5}\right)$ & $\left(8.70 \times 10^{-5}\right)$ \\
$15.50 \times 10^{-5}$ & $19.59 \times 10^{-5}$ & $31.14 \times 10^{-5}$ \\
$\left(8.34 \times 10^{-5}\right)$ & $\left(8.84 \times 10^{-5}\right)$ & $\left(9.93 \times 10^{-5}\right)$
\end{tabular}


Example 7. Lower tolerance limits for Data Set II, based on two Weibull models with increasing failure rates, are given in Table VII. The corresponding estimates of $\theta$ are $\hat{\theta}_{1.5}=6.73 \times 10^{-5}$ and $\hat{\theta}_{4}=2.53 \times$ $10^{-5}$. The Weibull distribution with $\alpha=4$ is extremely peaked, so that although the estimate of the central part of the distribution (including the tenth percentile) is decreasing with $\alpha$, the lower $(P, \gamma)$ tolerance bounds are increasing in $\alpha$ for the more extreme values of $P$.

In these examples we have made some rather arbitrary choices for the shape parameters, which might be based on our judgement about whether the failure rate is increasing or decreasing, and how fast. It might be preferable to estimate the shape parameter along with the scaie parameter. Unfortunately, such two-parameter models involve less commonly tabuiated distributions, if they are tractable at al1.

The distribution of $U=\hat{\alpha} \ln \left(\hat{\xi}_{p} / \xi_{p}\right)$, where $\hat{\alpha}$ and $\hat{\xi}_{p}$ are maximum likelihood estimates of the shape parameter $\alpha$ and the $p^{\text {th }}$ quantile $\xi_{p}$ of the Weibull distribution, turns out to be independent of the true values of the parameters $\alpha$ and $\theta$. In theory, therefore, toierance limits for the two-parameter Weibull distribution could be tabulated for various combinations of $P, \gamma, n$, and $r$. In practice, because the distribution of $U$ can only be generated by Monte Carlo techniques, these tables do not exist. However, it is perhaps interesting to note that for Data Set I, maximum likelihood estimation of two parameters for the Weibull distribution gives

$$
\hat{\alpha}=614, \hat{\theta}=11.19 \times 10^{4} \text {. }
$$

This estimated distribution has a decreasing failure rate. A $98 \%$ upper confidence bound on $\alpha$, derived from Table 1 in Ref. 5, is .874 . For Data Set II, estimates of the two parameters are

$$
\hat{\alpha}=2.37, \hat{\theta}=1.50 \times 10^{-5} \text {, }
$$

which lies between the two values used in Example 7. Tables for confidence bounds on the parameters are not available for this censored case, but as data are ivailable for only the early part of the distribution we cannot be very confident in our estimate of the shape of the tail. 
TABLE VII

LOWER TOLERANCE BOUNDS FOR DATA SET II BASED ON THE WEIBULL MODEL WITH SHAPE PARAMETER $\alpha=1.5(\alpha=4.0)$

\begin{tabular}{|c|c|c|c|}
\hline $\mathrm{P}$ & 0.90 & 0.95 & 0.99 \\
\hline 0.90 & $\begin{array}{c}2.62 \times 10^{4} \\
\left(1.98 \times 10^{4}\right)\end{array}$ & $\begin{array}{c}2.45 \times 10^{4} \\
\left(1.94 \times 10^{4}\right)\end{array}$ & $\begin{array}{c}2.18 \times 10^{4} \\
\left(1.85 \times 10^{4}\right)\end{array}$ \\
\hline 0.95 & $\begin{array}{c}1.62 \times 10^{4} \\
\left(1.66 \times 10^{4}\right)\end{array}$ & $\begin{array}{l}i .52 \times 10^{4} \\
\left(1.62 \times 10^{4}\right)\end{array}$ & $\begin{array}{c}1.35 \times 10^{4} \\
\left(1.55 \times 10^{4}\right)\end{array}$ \\
\hline 0.99 & $\begin{array}{c}0.55 \times 10^{4} \\
\left(1.10 \times 10^{4}\right)\end{array}$ & $\begin{array}{c}0.51 \times 10^{4} \\
\left(1.08 \times 10^{4}\right)\end{array}$ & $\begin{array}{c}0.45 \times 10^{4} \\
\left(1.03 \times 10^{4}\right)\end{array}$ \\
\hline
\end{tabular}

One other common two-parameter model for non-negative random variables is the lognormal distribution. Tolerance limits under this model are derived from tolerance limits for the normal distribution, and are of the form

$$
\begin{aligned}
& L \underset{\sim}{(X)}=\exp (\bar{Y}-K(n, P, Y ; S), \\
& U(\underset{\sim}{X})=\exp (\bar{Y}+K(n, P, Y) S) .
\end{aligned}
$$

Here $\bar{Y}$ is the average value of the logarithms of the (uncensored) observations and $S$ is the usual estimator of the standard deviation,

$$
s^{2}=\frac{1}{n-1} \sum_{i=1}^{n}\left[\log \left(x_{i}\right)-\bar{y}\right]^{2} .
$$

The factors $K(n, P, Y)$ are tabulated for several values of $n, P$ and $\gamma$ in Ref. 4 . Example 8. For Data Set $I, \bar{Y}=-12.399$ and $S=1.577$. Upper tolerance limits based on the lognormal distribution are shown in Table VIII. Although the lognormal distribution does not have a strictly decreasing failure rate, it is a "heavy-tailed" distribution, which results in large values for the upper tolerance limits when $P$ and $\gamma$ are 1 arge. 
TABLE VIII

UPPER TOLERANCE BOUNDS FOR DATA SET I BASED ON THE LOGNORMAL MGDEL

\begin{tabular}{c|ccc}
$\because Y$ & 0.90 & 0.95 & 0.99 \\
\hline 0.75 & $2.58 \times 10^{-5}$ & $3.35 \times 10-5$ & $6.03 \times 10-5$ \\
0.90 & $8.62 \times 10^{-5}$ & $12.32 \times 10-5$ & $28.07 \times 10^{-5}$ \\
0.95 & $18.22 \times 10^{-5}$ & $27.76 \times 10-5$ & $73.79 \times 10^{-5}$
\end{tabular}

IV. TOLERANCE LIMITS FOR DISTRIBUTIONS WITH MONOTONE FAILURE RATES

The exponential distribution forms a boundary between the distributions with increasing failure rates and those with decreasing failure rates. It is not surprising, therefore, that it is possible to derive tolerance limits, assuming only that the sampling distribution has a monotone failure rate, which resemble those for an exponential distribution. In particular, Barlow and Proschan (Ref. 6 ) show that if $F$ has an increasing failure rate, then

$$
\operatorname{Pr}\left\{\frac{C_{\gamma_{2} P, r}}{\hat{\theta}} \leq \xi_{1-P}\right\} \geq r,
$$

where

$$
c_{Y, P, r}=\min \left\{\frac{r}{n},-\frac{2 r \ln (P)}{x_{2 r, \gamma}^{2}}\right\}
$$

and $\hat{\theta}$ is given by Eq. (3.1).

Example 9. For Data Set II, $r / n=0.0373$, which is smaller than - $2 r \frac{\ln (P) / x_{2 r, \gamma}^{2}}{2}$ for $P=0.90$ and $\gamma=0.90$, but larger for $P=0.95$ and $P=0.99$. Therefire; lower $(0.90, \gamma)$ tolerance limits computed under the assumption of an increasing failure rate are $0.0373 / \hat{\theta}=1.65 \times 10^{4}$ for $\gamma=$ $0.90,0.95$, or 0.99 . However, the $(0.95, \gamma)$ and $(0.99, \gamma)$ tolerance limits remain as in Table IV.

v. BAYESIAN TOLERANCE LIMITS

In order to compute Bayesian tolerance limits it is necessary to formulate our initial state of knowledge in terms of a prior distribution on the 16 
parameters of the sampling distribution $F$. For the three one-parameter distributions of Sec. III, a convenient form for the prior distribution (the so-called "conjugate" prior) of the scale parameter $\theta$ is a gama distribution, with density

$$
\pi(\theta)=\frac{b^{a} \theta^{a-1} e^{-b \theta}}{\Gamma(a)}, \theta>0 .
$$

Therefore, we must use our initial knowledge to choose a shape parameter a and a scale parameter $b$ for this distribution. Following Ref. 7, we will do this using percentiles of the prior distribution.

When the sampling distribution is an exponential distribution with parameter $\theta$ and the prior distribution for $\theta$ is a gamma distribution with a half-integer shape parameter $a$ and scale parameter $b$, then posterior $(P, \gamma)$ tolerance limits are given by Eqs. (A.26) and (A.27), where $r$ is the number of uncensored observations and

$$
z=\sum_{i=1}^{r} x_{(i)}+(n-r) x_{(r)}
$$

For the prior $(P, Y)$ tolerance limits take $z=r=0$ in the same equations.

Example 10. In the exponential model for Data Set $I, \theta$ is just the expected lifetime, in hours, for a pump. Suppose that we feel the prior probability that time is less than three years is about 0.05 , while the probability that it is less than twenty years is 0.95 . A gamma distribution with $a=3.5$ and $b=4 \times 10^{-5}$ has its fifth quantile at $2.71 \times 10^{4}$ hours $=$ 3.09 years and ninty-fifth quantile at $17.58 \times 10^{4}$ hours $=20.07$ years, so this appears to reflect our beliefs adequately. Here $z=1.9814 \times 10^{-4}$, so $a+r=16.5$ and $z+b=2.3814 \times 10^{-4}$ are the parameters of the posterior gamma distribution. The posterior tolerance limits shown in Table IX are slightiy smaller than the classical limits of Table III.

Example 11. In the exponential model for Data Set II, $\theta$ is the failure rate, and $1 / \theta$ is the expected lifetime of the valves, in hours. A gamma distribution with $a=1.5$ and $b=1.7 \times 10^{5}$ has its fifth quantile at $\theta=$ $1.04 \times 10^{-6}$ failures per hour, corresponding to an expected lifetime of 110 
years, and its ninety-fifth quantile at $\theta=2.30 \times 10^{-5}$ failures per hour, corresponding to an expected lifetime of about five years. Taking this as our prior distribution, the posterior distribution has parameters $a+r=11.5$ and $b+z=4.604 \times 10^{6}$. Again, the posterior distribution has slightly smaller lower tolerance bounds (see Table $X$ ) than the classical results (Table IV).

\section{TABLE IXX}

POSTERIOR (PRIOR) UPPER TOL_ERANCE LIMITS FOR DATA SET I BASED ON AN EXPONENTIAL SAMPLING DISTRIBUTION AND A GAMMA PRIOR WITH

\begin{tabular}{l|crr}
$\ddots \gamma$ & 0.90 & 0.95 & 0.99 \\
\hline 0.75 & $2.86 \times 10^{-5}$ & $3.16 \times 10^{-5}$ & $3.87 \times 10^{-5}$ \\
$\left(3.91 \times 10^{-5}\right)$ & $\left(5.12 \times 10^{-5}\right)$ & $\left(8.95 \times 10^{-5}\right)$ \\
0.90 & $\begin{array}{l}3.75 \times 10^{-5} \\
4.95\end{array}$ & $5.26 \times 10^{-5}$ & $6.42 \times 10^{-5}$ \\
$\left(6.50 \times 10^{-5}\right)$ & $\left(8.50 \times 10^{-5}\right)$ & $\left(14.87 \times 10^{-5}\right)$ \\
$6.17 \times 10^{-5}$ & $6.84 \times 10^{-5}$ & $8.36 \times 10^{-5}$ \\
$\left(8.46 \times 10^{-5}\right)$ & $\left(11.06 \times 10^{-5}\right)$ & $\left(19.34 \times 10^{-5}\right)$
\end{tabular}

TARLE X

POSTERIOR (PRIOR) LOWER TOLERANCE LIMITS FOR DATA SET II BASED ON AN EXPONENTIAL SAMPLING DISTRIBUTION AND A GAMMA PRIOR WITH $a=1.5, b=1.7 \times 10^{5}$

\begin{tabular}{l|ccr}
$Y Y$ & & & \\
& 0.90 & 0.95 & 0.99 \\
\hline 0.90 & $3.03 \times 10^{4}$ & $2.76 \times 10^{4}$ & $2.33 \times 10^{4}$ \\
$\left(0.57 \times 10^{4}\right)$ & $\left(0.46 \times 10^{4}\right)$ & $\left(0.32 \times 10^{4}\right)$ \\
0.95 & $1.48 \times 10^{4}$ & $1.34 \times 10^{4}$ & $1.13 \times 10^{4}$ \\
$\left(0.28 \times 10^{4}\right)$ & $\left(0.22 \times 10^{4}\right)$ & $\left(0.15 \times 10^{4}\right)$ \\
0.99 & $\left(0.29 \times 10^{4}\right)$ & $0.26 \times 10^{4}$ & $0.22 \times 10^{4}$ \\
& $\left(0.055 \times 10^{4}\right)$ & $\left(0.044 \times 10^{4}\right)$ & $\left(0.030 \times 10^{4}\right)$
\end{tabular}


When the sampling distribution is a gamma distribution with known, half-integer shape parameter $\alpha$, and the prior distribution for the scale parameter $\theta$ is a gamma distribution with parameters a (also a half-integer) and $b$, then the posterior upper $(P, \gamma)$ tolerance limit is given by Eq. (A.29). The prior tolerance limit uses $n=z=0$ in that eauation.

Example 12. For Data Set I, take $\alpha=0.5$ and use the prior distribution of Example $8\left(a=3.5, b=4 \times 10^{-5}\right)$, although $\theta$ can no longer be interpreted as the expected lifetime of the pumps. The posterior gama distribution has parameters $n \alpha+a=10$ and $z+b=4.604 \times 10^{6}$. The posterior upper tolerance limits of Table $X I$ are about twice as large as the corresponding $c l$ assical limits in Table $V$.

When the sampling distribution is a Weibull distribution with known shape parameter, it is easy to work with a prior gamma distribution on $\beta=\theta^{\alpha}$. If a and $b$ are the parameters of this distribution, then the prior lower $(P, Y)$

tolerance limit is given by Eq. (A.30), where $z=\sum_{i=1}^{r} x_{(i)}^{\alpha}+(n-r) x_{(r)}^{\alpha}$.

TABLE XI

POSTERIOR (PRIOR) UPPER TOLERANCE LIMITS FOR DATA SET I BASED ON A GAMMA SAMPLING DISTRIBUTION WITH SHAPE FARAMETER 0.5 AND A GAMMA PRIOR

WITH $a=3.5, b=4 \times 10^{-5}$

\begin{tabular}{c|ccc}
0.75 & 0.90 & 0.95 & 0.99 \\
\hline 0.90 & $\begin{array}{l}3.00 \times 10^{-5} \\
\left(1.87 \times 10^{-5}\right)\end{array}$ & $\left(2.44 \times 10^{-5}\right)$ & $12.32 \times 10^{-5}$ \\
$13.24 \times 10^{-5}$ & $16.35 \times 10^{-5}$ & $25.19 \times 10^{-5}$ \\
$\left(3.82 \times 10^{-5}\right)$ & $\left(4.99 \times 10^{-5}\right)$ & $\left(8.73 \times 10^{-5}\right)$ \\
$18.80 \times 10^{-5}$ & $23.22 \times 10^{-5}$ & $35.76 \times 10^{-5}$ \\
$\left(5.42 \times 10^{-5}\right)$ & $\left(7.09 \times 10^{-5}\right)$ & $\left(12.40 \times 10^{-5}\right)$
\end{tabular}


Example 13. In order to develop a prior distribution for $\beta$, note that it is related to the reliability function $R(x)=1-F(x)$ by

$$
R(x)=\exp \left(-\beta x^{\alpha}\right)
$$

or

$$
B=-\frac{\ln (R(x))}{x^{a}}
$$

Suppose our prior knowledge about the type of valves in Data Set II can be expressed in terms of their reliability at $10^{5}$ hours (about 11.4 years). Specifically, suppose we believe that

$$
\operatorname{Pr}\left\{R\left(10^{5}\right) \geq 0.9\right\}=0.05
$$

and

$$
\operatorname{Pr}\left\{R\left(10^{5}\right) \geq 0.1\right\}=0.95
$$

Using $\alpha=1.5$, this implies two percentiles for the prior distribution of $\beta$,

$$
\operatorname{Pr}\left\{\beta \leq 3.33 \times 10^{-9}\right\}=0.05,
$$

and

$$
\operatorname{Pr}\left\{\beta \leq 7.28 \times 10^{-8}\right\}=0.95
$$

A gamma distribution with $a=1.5$ and $b=5.3 \times 10^{7}$ comes close to meeting these requirements, with

$$
0.05=\operatorname{Pr}\left\{B \leq 3.32 \times 10^{-9}\right\}=\operatorname{Pr}\left\{R\left(10^{5}\right) \geq 0.097\right\}
$$




$$
0.95=\operatorname{Pr}\left\{\beta \leq 7.37 \times 10^{-8}\right\}=\operatorname{Pr}\left\{R\left(10^{5}\right) \geq 0.9004\right\} .
$$

For the posterior distribution we then have $a+r=11.5$ and $b+z=$ $6.26 \times 10^{8}$. The posterior tolerance limits shown in Table XII are very close to the classical limits for the Weibull model in Table VII.

Table XIII shows the effect of a different prior distribution (a $=4.5$, $\left.b=3.27 \times 10^{9}\right)$, corresponding to

$$
\operatorname{Pr}\left\{R\left(10^{5}\right) \geq 0.99\right\}=0.01
$$

and

$$
\operatorname{Pr}\left\{R\left(10^{5}\right) \geq 0.90\right\}=0.99
$$

This more precise and optimistic prior specification of the reliability at $10^{5}$ hours results in posterior tolerance limits which are three times as 1 arge as those of Table XII.

TABLE XII

POSTERIOR (PRIOR) LOWER TOLERANCE LIMITS FOR DATA SET II BASED ON A WEIBULL SAMPLING DISTRIBUTION WITH SHAPE PARAMETER 1.5 AND A GAMMA PRIOR WITH $a=1.5, b=5.3 \times 10^{7}$

\begin{tabular}{c|ccc} 
& & & \\
0.90 & 0.90 & 0.95 & 0.99 \\
\hline \multirow{1}{*}{0.95} & $2.57 \times 10^{4}$ & $2.41 \times 10^{4}$ & $2.16 \times 10^{4}$ \\
& $\left(1.47 \times 10^{4}\right)$ & $\left(1.27 \times 10^{4}\right)$ & $\left(.99 \times 10^{4}\right)$ \\
$1.59 \times 10^{4}$ & $1.49 \times 10^{4}$ & $1.34 \times 10^{4}$ \\
$\left(0.91 \times 10^{4}\right)$ & $\left(0.79 \times 10^{4}\right)$ & $\left(0.61 \times 10^{4}\right)$ \\
& $0.54 \times 10^{4}$ & $0.50 \times 10^{4}$ & $0.45 \times 10^{4}$ \\
$\left(0.31 \times 10^{4}\right)$ & $\left(0.26 \times 10^{4}\right)$ & $\left(0.21 \times 10^{4}\right)$
\end{tabular}


TABLE XIII

\begin{tabular}{|c|c|c|c|}
\hline \multicolumn{4}{|c|}{$\begin{array}{l}\text { POSTERIOR } \\
\text { WEIBULL }\end{array}$} \\
\hline$P$ & 0.90 & 0.95 & 0.99 \\
\hline 0.90 & $\begin{array}{r}7.54 \times 10^{4} \\
\left(13.01 \times 10^{4}\right)\end{array}$ & $\begin{array}{r}7.13 \times 10^{4} \\
\left(11.84 \times 10^{4}\right)\end{array}$ & $\begin{array}{r}6.44 \times 10^{4} \\
\left(10.04 \times 10^{4}\right)\end{array}$ \\
\hline 0.95 & $\begin{array}{r}4.67 \times 10^{4} \\
\left(8.05 \times 10^{4}\right)\end{array}$ & $\begin{array}{r}4.41 \times 10^{4} \\
\left(7.33 \times 10^{4}\right)\end{array}$ & $\begin{array}{c}3.98 \times 10^{4} \\
\left(5.21 \times 10^{4}\right)\end{array}$ \\
\hline 0.99 & $\begin{array}{r}1.57 \times 10^{4} \\
\left(2.72 \times 10^{4}\right)\end{array}$ & $\begin{array}{c}1.49 \times 10^{4} \\
\left(2.47 \times 10^{4}\right)\end{array}$ & $\begin{array}{c}1.34 \times 10^{4} \\
\left(2.10 \times 10^{4}\right)\end{array}$ \\
\hline
\end{tabular}

Upper Bayesian tolerance limits for the two-parameter lognormal mode? take the form

$$
U=\exp \left\{a+\sqrt{\frac{b}{N-1}} K(N, P, \gamma)\right\},
$$

where $K(N, F, Y)$ are the factors which were used in the classical lognormal tolerance limits, and $a, N$ and $b$ are the parameters of the prior or posterior normal-gama distribution for the parameters $(\mu, \sigma)$ of the sampling distribution (see Eqs. (A.35) and (A.36)). The parameters are given by

$$
\begin{aligned}
& a=\bar{Y}_{0} \\
& N=n_{0} \\
& b=\left(n_{0}-1\right) s_{0}{ }^{2}
\end{aligned}
$$

for the prior distribution, and 


$$
\begin{aligned}
& a=\frac{{ }^{n} 0^{\bar{Y}}+n \bar{Y}}{n_{0}+n} \\
& N=n_{0}+n \\
& b=\left(n_{0}-1\right) s_{0} S^{2}+(n-1) S^{2}+\frac{n_{0} n}{n_{0}+n}\left(\bar{Y}-\bar{Y}_{0}\right)^{2}
\end{aligned}
$$

for the posterior distribution. Thus, $y_{0}$ and $\mathrm{S}_{0}{ }^{2}$ should represent our initial knowledge about the parameters of the lognormal sampling distribution, and $n_{0}$ (by comparison with $n$ ) suggests how we wish to weight this prior knowledge compared to the data.

Example 14. For the pump failure rate data, suppose we expect the mean and variance of the $\log$ failure rates to be about $\gamma_{0}=-10$. and $s_{0}^{2}=1$., and let us give almost as much weight to this as to the data $\left(n_{0}=10.\right)$ for the prior distribution, then,

$$
\begin{aligned}
& a=-10 . \\
& N=10 \\
& b=9 .
\end{aligned}
$$

For the posterior distribution, because $n=13, \bar{Y}=-12.399$ and $S^{2}=$ 2.48693, we get

$$
\begin{aligned}
& a=-11.356, \\
& N=23 \\
& b=7.13726 .
\end{aligned}
$$

Table XIV shows the resulting upper tolerance limits. Despite our rather pessimistic prior, the posterior tolerance limits are much smaller than the classical ones (Table VIII).

VI. SAMPLE SIZE AND ACCURACY

In Sec. II we observed that nonparametric $(P, Y)$ tolerance limits can be computed only if the sample size $n$ is sufficiently large that

$$
p^{n} \leq 1-\gamma \text {. }
$$

Minimum values for $n$ as a function of $P$ and $Y$ are shown in Table XV. 
TABLE XIV

POSTERIOR (PRIOR) UPPER TOLERANCE LIMITS FOR DATA SET I BASED ON A LOGNORMAL SAMPLING DISTRIBUTION WITH AN NI $(-10 ., 10,9$.$) PRIOR$
'Y
$P .75$
0.90
0.95
0.99
$2.09 \times 10^{-5}$
$3.12 \times 10^{-5}$
$4.00 \times 10^{-5}$
$\left(15.96 \times 10^{-5}\right)$
$\left(19.65 \times 10^{-5}\right)$
$\left(32.04 \times 10^{-5}\right)$
0.90
$2.22 \times 10^{-5}$
$3.39 \times 10^{-5}$
$4.41 \times 10^{-5}$
$\left(35.80 \times 10^{-5}\right)$
$\left(47.84 \times 10^{-5}\right)$
$\left(95.67 \times 10^{-5}\right)$
0.95
$2.53 \times 10^{-5}$
$4.05 \times 10^{-5}$
$5.42 \times 10^{-5}$
$\left(59.20 \times 10^{-5}\right)$
$\left(83.42 \times 10^{-5}\right)$
$\left(190.93 \times 10^{-5}\right)$

Under the parametric models of Sec. III, $(P, Y)$ tolerance limits are available for any $P$ and $\gamma$, provided that we have enough observations (one or more) to estimate the unknown parameters. That is, under these models a statistic $L(\underset{\sim}{X})$ (or $U(\underset{\sim}{X})$ ) is always available such that

$$
\begin{gathered}
\operatorname{Pr}\left\{\left(\underset{\sim}{X)} \leq \xi_{1-p}\right\}=\gamma\right. \\
\text { (or } \left.\operatorname{Pr}\left\{\underset{\sim}{U(X)} \geq \xi_{p}\right\}=\gamma\right),
\end{gathered}
$$

TABLE XV

MINIMUM SAMPLE SIZES FOR NONPARAMETRIC $(P, \gamma)$ TOLERANCE LIMITS

\begin{tabular}{r|r}
$P Y \gamma$ & 0.90 \\
\hline 0.75 & 9 \\
0.90 & 22 \\
0.95 & 45 \\
0.99 & 230
\end{tabular}

0.95

0.99

11

17

29

44

59

90

299

459 
provided that the model is in fact correct. However, the size of the sample does affect the accuracy with which the $p^{\text {th }}$ quantile can be estimated.

Several authors (Refs. 8 and 9) have proposed as a measure of accuracy, not the distance between $\xi_{1-P}$ and $L(\underset{\sim}{X})$ (or between $\xi_{P}$ and $U(\underset{\sim}{X})$ ), but rather the difference between $F(L(\underset{\sim}{X})$ ) and $1-P$ (or between $F(U(\underset{\sim}{X})$ ) and $P$.) This is more appropriate in the context of tolerance intervals. Specifically, from the definition of a lower $(P, \gamma)$ tolerance limit $L(\underset{\sim}{X})$, we know that

$$
\operatorname{Pr}\{1-F(L(\underset{\sim}{X})) \geq P\}=\gamma .
$$

The question is, how 1 arge a sample must we have to guarantee that, in addition,

$$
\operatorname{Pr}\{1-F(L(\underset{\sim}{X})) \geq P+\Delta P\} \leq 1-\gamma ?
$$

The answer to this question clearly will depend on $P, \Delta P$, and $\gamma$.

For any Weibull model with known shape parameter (includiny the expenential with shape parameter $\alpha=1$ ), Eqs. (6.1) and (6.2) can be satisfied simultaneously provided that the number of uncensored observations $r$ is sufficiently large that

$$
\frac{x_{2 r, \gamma}^{2}}{x_{2 r, 1-\gamma}^{2}} \leq \frac{\ln (P)}{\ln (P+\Delta P)} .
$$

A similar result is obtained for upper tolerance limits. If $U(\underset{\sim}{X})$ satisfies

$$
\operatorname{Pr}\{F(\underset{\sim}{(\mathcal{X})})) \geq P\}=\gamma,
$$

then in order to have, in addition,

$$
\operatorname{Pr}\{F(\underset{\sim}{(\underset{X}{X}))} \geq P+\Delta P\} \leq 1-\gamma,
$$

we require 


$$
\frac{x_{2 r, \gamma}^{2}}{x_{2 r, 1-\gamma}^{2}} \leq \frac{\ln (1-P-\Delta P)}{\ln (1-P)}
$$

Among other things, the problem is no longer symetric for lower and upper tolerance limits (as it was for the nonparametric case.) As Tables XVI and XVII show, larger samples are required for the accurate estimation of upper tail percentiles than for the lower end of the distribution.

A glance at Tables XVI and XVII shows that for the samples in our two data sets $(r=13$ and $r=10$, respectively) the inequalities (6.2) and (6.5) certainly do not hold. In fact, for a sample of size thirteen, the

\section{TABLE XVI}

MINIMUM SAMPLE SIZES TO OBTAIN LOWER $(P, \gamma)$ TOLERANCE LIMITS SUCH THAT $\operatorname{Pr}\{1-F(L) \geq P+\triangle P\} \leq 1-\gamma$ UNDER THE WEIBULL MODEL WITH KNOWN SHAPE PARAMETER

\begin{tabular}{l|l|rrr}
$P$ & $\Delta P$ & $Y=0.90$ & 0.95 & 0.99 \\
\hline 0.90 & 0.025 & 73 & 120 & 240 \\
& 0.01 & 536 & 883 & 1765 \\
0.95 & 0.025 & 14 & 23 & 44 \\
& 0.01 & 127 & 208 & 416
\end{tabular}

TABLE XVII

MINIMUM SAMPLE SIZES TO OBTAIN UPPER ( $P, \gamma$ ) TOLERANCE LIMITS SUCH THAT $\operatorname{Pr}\{F(U) \geq P+\triangle P\} \leq 1-Y$

UNDER THE WEIBULL MODEL WITH KNOWN SHAPE PARAMETER

\begin{tabular}{|c|c|c|c|c|}
\hline$P$ & $\Delta P$ & $Y=0.90$ & 0.95 & 0.99 \\
\hline \multirow[t]{2}{*}{0.90} & 0.025 & 475 & 782 & 1563 \\
\hline & 0.01 & 3283 & 5407 & 10815 \\
\hline 0.95 & 0.025 & 153 & 251 & 501 \\
\hline & 0.01 & 1274 & 2098 & 4195 \\
\hline
\end{tabular}


probability that a $(0.90,0.95)$ upper tolerance limit computed under a Weibul? model is actually greater than $\xi_{0.95}$ is 0.79 , and it has probability .24 of being greater than $\xi_{0.99}$. A lower $(0.95,0.95)$ tolerance limit based on ten uncensored observations has probability 0.25 of being less than $\xi_{0.025}$. Thus, tolerance limits based on small samples are likely to be very conservative.

Similar results are obtained for tolerance limits estimated under the gamma model with known shape parameter. There is some dependency on the shape parameter; the sample size required for given accuracy of lower tolerance limits increases with $\alpha$, while that required for upper tolerance 1 imits decreases. The details are given in App. B.

For Bayesian tolerance limits, the sample size required for given accuracy depends, of course, on the choice of a prior distribution. The more precise our prior knowlenge, the fewer the number of observations required. For the exponential distribution with a garma prior, for example, the number of observations $r$ must be such that $a+r$ is as large as the number given in Table XVI and XVII, where $a$ is the shape parameter of the garma prior. We saw in the examples of Sec. $V$ that reasonable priors corresponded to rather small values of $a$, so the sample size requiremerits are not much different here than from those for classical tolerance limits. Very similar conclusions hold for the other Bayesian models considered in Sec. V.

\section{CONCLUSIONS}

In Sec. VI, we saw that the direct effect of small sample sizes is to make tolerance limits conservative. By definition, an upper $(P, \gamma)$ tolerance limit has probability $\gamma$ of bounding a fraction $P$ or more of the population from above. When this statistic is based on small samples, the probability of bounding a larger fraction $P+\Delta P$ of the population is substantial.

However, a far more serious effect, which can easily overwhelm this tendency towards conservatism, is the effect which the choice of a statistical model for the observations has on the computed tolerance limits. By way of review, the upper $(0.90,0.95)$ tolerance limits for Data Set I which were computed in Secs. III and $V$ are sumarized in Table XVIII. It will be seen that these vary by a factor of four, from $3.39 \times 10^{-5}$ to $16.35 \times 10^{-5}$. A factor of four can invalidate our conclusions. Suppose, for example, that we were to use the exponential model (a very common choice when only a few observations are available.) If the observations actually come from a 
TABLE XVIII

SUMMARY OF PARAMETRIC UPPER $(0.90,0.95)$ TOLERANCE LIMITS FOR DATA SET I

\begin{tabular}{llr} 
& \multicolumn{1}{c}{ Model } & Tolerance Limit \\
\cline { 2 - 3 } & exponential & $5.93 \times 10^{-5}$ \\
Classical & gamma, $\alpha=0.5$ & $9.10 \times 10^{-5}$ \\
& gama, $\alpha=2.0$ & $4.23 \times 10^{-5}$ \\
& Weibuil, $\alpha=0.5$ & $11.57 \times 10^{-5}$ \\
& Weibuil, $\alpha=2.0$ & $7.75 \times 10^{-5}$ \\
& lognormal & $12.32 \times 10^{-5}$ \\
Bayesian & exponential & $5.26 \times 10^{-5}$ \\
& gamma, $\alpha=0.5$ & $16.35 \times 10^{-5}$ \\
& lognormal & $3.39 \times 10^{-5}$
\end{tabular}

heavier-tailed distribution with $\xi_{0.90}$ up around $9 \times 10^{-5}$ (which seems possible in view of the tolerance limits computed using decreasing failure rate mode $1 \mathrm{~s})$, then our computed $(0.90,0.95)$ upper tolerance limit, which was $5.93 \times 10^{-5}$ under the exponential model, may not bound more than 50 or $60 \%$ of the population. This result is anything but conservative.

This is, of course, a perennial statistical difficulty, and perhaps more a philosophical problem than a technical one. However, it is hoped that this series of examples will encourage the analyst to exercise as much care as possible in the selection of statistical models and : bear in mind that the final result is only as valid as the assumptions used to relect the method of analysis.

\section{REFERENCES}

1. W.J. Conover, Practical Nonparametric Statistics (John Wiley and Sons, Inc., New York, 1971), pp. 116-120.

2. N.L. Johnson and S. Kotz, Continuous Univariate Distributions - 1 (Houghton Mifflin Company, Boston, 1970).

3. N.R. Mann, R.E. Schafer, and N.D. Singpurwalla, Methods for Statistical Analys is of Reliability and Life Data (John Wiley and Sons, Inc. New York, 1974).

4. A.H. Bowker and G.J. Lieberman, Engineering Statistics (Prentice-Hall, Inc., Englewood Cliffs, NJ, 1972), pp. 314-315.

5. D.R. Triuinan, L.J. Bain, and C.E. Antle, "Inferences on the Parameters of the Weibu11 Distribution," Technometrics 11, 445-460 (1969). 
6. R.E. Bar low and F. Proschan, "Tolerance and Confidence Limits for Classes of Distributions Based on Failure Rate," Ann. Math. Stat. 37, 1593-1601 (1966).

7. R.A. Watler, M.M. Johnson, M.S. Waterman, and H.F. Martz, Jr., "Ganma Prior Distribution Selection for Bayesian Analys is of Failure Rate and Reliability," presented at the International Conference on Nuclear Systems Reliability Engineering and Risk Assessment, Los Alamos Scientific Laboratory report LA-UR-77-155 (1977).

8. G.D. Faulkenberry and P.L. Weeks, "Sample Size Determination for Tolerance Limits," Technometrics 10, 343-348 (1968).

9. R.L. Kirkpatrick, "Sample Sizes to Set Tolerance Limits," J. Quality Technology $\underline{9}, 6-12$ (1977).

\section{APPENDIX A}

\section{DERIVATION OF TOLERANCE LIMITS FOR SELECTED PARAMETRIC MODELS}

I. ONE-SIDED TOLERANCE LIMITS FOR ONE-PARAMETER MODELS

Suppose that the observations $x_{1}, X_{2}, \ldots$ are drawn from an unknown member of a family of distributions indexed by a single parameter $\theta$. In this case, the $p^{\text {th }}$ quantile of a member of the family is specified once $\theta$ is known, and we write it explicitly as $\xi_{p}(\theta)$.

Let $\hat{\theta}(X)$ be an estimator for $\theta$. Denote the one-sided $100 \%$ confidence bounds fi: $\tilde{\theta}$, based on $\hat{\theta}(\underset{\sim}{X})$, by $\underline{\theta}_{\gamma}(\underset{\sim}{X})$ and $\bar{\theta}_{\gamma}(\underset{\sim}{X})$. If $\xi_{p}(\theta)$ is a monotonic increasing function of $\theta$, then

$$
\operatorname{Pr}\left\{\xi_{p}\left(\bar{\theta}_{\gamma}(X)\right) \geq \xi_{p}(\theta)\right\}=\operatorname{Pr}\left\{\bar{\theta}_{\gamma}(X) \geq \theta\right\}=\gamma,
$$

and thus, $\underset{\sim}{U} \underset{\sim}{X})=\xi_{P}\left(\bar{\theta}_{\gamma}(\underset{\sim}{X})\right)$ is an upper $(P, \gamma)$ tolerance limit for $F_{\theta}$, whatever the true value of $\theta$. Similarly,

$$
L(\underset{\sim}{x})=\xi_{1-p}\left(\underline{\theta}_{\gamma}(\underset{\sim}{x})\right)
$$

is a lower $(P, \gamma)$ tolerance limit. On the other hand, when $\xi_{p}(\theta)$ and $\xi_{1-p}(\theta)$ are decreasing functions of $\theta$ (as they will be in all of the examples of this section), we use 


$$
\begin{aligned}
& L(\underset{\sim}{x})=\xi_{1-p}\left(\bar{\theta}_{\gamma}(\underline{x})\right) \\
& U(\underset{\sim}{x})=\xi_{p}\left(\underline{\theta}_{\gamma}(\underset{\sim}{x})\right) .
\end{aligned}
$$

Let $F_{\theta}$ denote the exponential distribution with positive parameter $\theta$,

$$
F_{\theta}(x)=\operatorname{Pr}\{x \leq x\}=1-e^{-\theta x}, x>0 .
$$

Let $\hat{\theta}$ be the maximum likelihood estimator for $\theta$ based on a Type II censored sample $x_{(1)}, \ldots, x_{(r)}$ where $1 \leq r \leq n$,

$$
\hat{\theta}=\frac{r}{\sum_{i=1}^{r} x_{(i)}+(n-r) x_{(r)}} .
$$

Then $2 r \theta / \hat{\theta}$ has a chi-square distribution with $2 r$ degrees of freedom (Ref. 3 , p. 165), giving $100 \%$ confidence bounds

$$
\begin{aligned}
& \underline{\theta}_{-\gamma}=\frac{\hat{\theta} \times 2 r, 1-\gamma}{2 r} \\
& \underline{\theta}_{\gamma}=\frac{\hat{\theta} \times 2 r, \gamma}{2 r} .
\end{aligned}
$$

The $P^{\text {th }}$ quantile of $F_{\theta}$ is the solution to the equation

$$
F_{\theta}(x)=1-e^{-\theta x}=P \text {, }
$$

which is

$$
\xi_{p}(\theta)=-\frac{\ln (1-P)}{\theta},
$$


a decreasing function of $\theta$. Combining Eqs. (A.3), (A.4), and (A.5) according to the rules of Eqs. (A.1) and (A.2) gives

$$
\begin{aligned}
& L \underset{\sim}{(X)}=-\frac{2 r \ln (P)}{\hat{\theta} x_{2 r, \gamma}^{2}} \\
& \underset{\sim}{U}(x)=-\frac{2 r \ln (1-P)}{\hat{\theta} x_{2 r, 1-\gamma}^{2}} .
\end{aligned}
$$

Next we consider the case where $F_{\theta}$ is a gamma distribution with known shape parameter $\alpha$ and unknown scale parameter $\theta$. The probability density function of $F_{\theta}$ is

$$
f_{\theta}(x)=\frac{\theta^{\alpha} x^{\alpha-1} e^{-\theta x}}{\Gamma(\alpha)}, \quad x>0
$$

If $x_{1}, \ldots x_{n}$ is an uncensored sample from this distribution, then $\sum_{i=1}^{n} x_{i}$

has a garma distribution with parameters $n \alpha$ and $\theta$. The maximum likelihood estimator for $\theta$ is

$$
\hat{\theta}=\frac{n \alpha}{\sum X_{i}}
$$

and $2 \operatorname{nov} \theta \hat{\theta}=2 \theta \sum_{i=1}^{n} x_{i}$ has a gamma distribution with parameters $n \alpha$ and $1 / 2$.

For arbitrary $\alpha$, we could use this fact to derive upper and lower confidence limits on $\theta$. However, if we assume that $\alpha=k / 2$ for some integer $k$, then we may use the fact that this gama distribution with parameters $n k / 2$ and $1 / 2$ is the chi-square distribution with $n k=2 n \alpha$ degrees of freedom. With this assumption we obtain

$$
\underline{\theta}_{\gamma}=\frac{\hat{\theta} x_{2 n \alpha, 1-\gamma}^{2}}{2 n \alpha}
$$




$$
\hat{\theta}_{\gamma}=\frac{\bar{\theta}_{\gamma} x_{2 n \alpha, \gamma}^{2}}{2 n \alpha}
$$

By definition, the $p^{\text {th }}$ quantile $\xi_{p}(\theta)$ satisfies the equation

$$
\frac{\theta^{\alpha}}{\Gamma(\alpha)} \int_{0}^{\xi_{p}(\theta)} t^{\alpha-1} e^{-\theta t} d t=p
$$

With a change of variables, $s=\theta t$, this equation becomes

$$
\frac{1}{\Gamma(\alpha)} \int_{0}^{\theta \xi_{p}(\theta)} s^{\alpha-1} e^{-s} d s=p
$$

which is true for all $\theta$ only if $\xi_{p}(\theta)$ is of the form $c / \theta$. The constant $c$ is determined by the fact that $\xi_{p}(1 / 2)=2 c$ must be the $p^{\text {th }}$ percentile of the gamma distribution with parameters $\alpha$ and $1 / 2$ or the chi-square distribution with $2 \alpha$ degrees of freedom. Thus

$$
\xi_{p}(\theta)=\frac{x^{2}}{2 \alpha, p}
$$

Equations (A.8), (A.9), and (A.10) combine to give the tolerance limits

$$
\begin{aligned}
& L(\underset{\sim}{x})=\frac{n \alpha x_{2 \alpha, 1-p}^{2}}{\hat{\theta} x_{2 n \alpha, \gamma}^{2}} \\
& \underset{\sim}{(x)}=\frac{n \alpha x_{2 \alpha, P}^{2}}{\hat{\theta} x_{2 n \alpha, 1-\gamma}^{2}} .
\end{aligned}
$$

If $F_{\theta}$ is a Weibull distribution with known shape parameter $\alpha$, then

$$
F_{\theta}(x)=\operatorname{Pr}\{x \leq x\}=1-\exp \left(-\theta^{\alpha} x^{\alpha}\right), x \geq 0
$$


It follows that $Y=\chi^{\alpha}$ has an exponential distribution with parameter $\beta=\theta^{\alpha}$, for

$$
\operatorname{Pr}\{y \leq y\}=\operatorname{Pr}\left\{x \leq y^{1 / \alpha}\right\}=1-\exp \left(-\theta^{\alpha} y\right) .
$$

The maximum likelihood estimator for $B$ is therefore

$$
\hat{B}=\frac{r}{\sum_{i=1}^{r} x_{(i)}^{\alpha}+(n-r) x_{(r)}^{\alpha}},
$$

and $2 r \beta / \hat{\beta}$ has a chi-square distribution with $2 r$ degrees of freedom, leading to confidence bounds like Eqs. (A.3) and (A.4), with $\theta$ replaced by $\beta$. Fur thermore,

$$
P=\operatorname{Pr}\left\{x \leq \xi_{p}(\theta)\right\}=\operatorname{Pr}\left\{y \leq \xi_{p}(\theta)^{\alpha}\right\}
$$

implies that

$$
\xi_{p}(\theta)^{\alpha}=-\frac{\ln (1-P)}{\theta^{\alpha}}=-\frac{\ln (1-P)}{\beta} .
$$

Thus, tolerance limits for this case are given by

$$
\begin{aligned}
& L(\underset{\sim}{X})=\left(-\frac{2 r \ln (P)}{\hat{\beta} X_{2 r, \gamma}^{2}}\right)^{1 / \alpha} \\
& U(\underset{\sim}{X})=\left(-\frac{2 r \ln (1-P)}{\hat{\beta} \chi_{2 r, 1-\gamma}^{2}}\right)^{1 / \alpha} .
\end{aligned}
$$

II. THE TWO-PARAMETER LOGNORMAL MODEL

If the $x_{i}$ come from a lognormal distribution, then $y_{i}=\ln x_{i}$ is normally distributed with parameters $\mu$ and $\sigma^{2}$. Sufficient statistics for this case are given by 


$$
\begin{aligned}
& \bar{Y}=\frac{1}{n} \sum_{i=1}^{n} Y_{i} \\
& s^{2}=\frac{1}{n-1} \sum_{i=1}^{n}\left(Y_{i}-\bar{Y}\right)^{2} .
\end{aligned}
$$

$Y$ and $S^{2}$ are independent of one another, $\sqrt{n}(\bar{Y}-\mu) / \sigma$ has a standard normal distribution, and $(n-1) s^{2} / \sigma^{2}$ has a chi-square distribution with $n-1$ degrees of freedom.

An upper $(P, Y)$ tolerance limit of the form $U(\underset{\sim}{Y})=\bar{Y}+K(n, P, Y) S$ is easily developed. By definition, we require

$$
\operatorname{Pr}\left\{\bar{\gamma}+K(n, P, \gamma) S \geq \xi_{p}\right\}=\gamma,
$$

where $\xi_{p}$ denotes the $p^{\text {th }}$ quantile of the $N\left(\mu, \sigma^{2}\right)$ distribution. This is related to the $P^{\text {th }}$ quantile $z_{p}$ of the standard normal by

$$
\xi_{p}=\mu+z_{p} \sigma
$$

Inserting this into Eq. (A.15) and rearranging we get finally

$$
\operatorname{Pr}\left\{\frac{-\sqrt{n} \frac{\bar{y}-\mu}{\sigma}+\sqrt{n z} p}{\sqrt{\frac{s^{2}}{\sigma 2}}} \leq \sqrt{n} k(n, P, \gamma)\right\}=\gamma .
$$

Now $-\sqrt{n}(\bar{Y}-\mu) / \sigma$ has a standard normal distribution, and $s^{2} / \sigma^{2}$ is independently distributed as a chi-square divided by its degrees of freedom, $n-1$. So the left side has a non-central $t$ distribation with $n$ - 1 degrees of freedom and non-centrality parameter $\sqrt{n} z_{p}$, and Eq. (A.16) implies that $\sqrt{n} K(n, P, \gamma)$ is the $\gamma^{\text {th }}$ quantile of this distribution,

$$
K(n, P, \gamma)=\frac{1}{\sqrt{n}} t_{n-1, \gamma}\left(\sqrt{n} z_{p}\right) .
$$


These factors $K$ have been tabulated (Ref. 4).

The $p^{\text {th }}$ quantile of the lognormal distribution of the $X_{i}$ 's is $\exp \left(\xi_{p}\right)$, and $\exp (\bar{Y}+K(n, P, \gamma) S)$ is a $100 \%$ upper confidence bound for $\exp \left(\xi_{p}\right)$. That is, an upper $(P, Y)$ tolerance bound for the two-parameter lognormal distribution is given by

$$
U(\underset{\sim}{X})=\exp (\bar{Y}+K(n, P, Y) S)
$$

A similar argument yields

$$
L(\underset{\sim}{X})=\exp (\bar{Y}-K(n, P, Y) S)
$$

III. BAYESIAN TOLERANCE LIMITS FOR ONE-PARAMETER MODELS

Bayesian $(P, Y)$ tolerance limits are random variables $L(\underset{\sim}{X})$ and $U(\underset{\sim}{X})$ for which

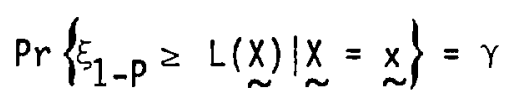

and

$$
\operatorname{Pr}\{\xi \mathrm{p} \leq U(\underset{\sim}{x}) \mid \underset{\sim}{x}=\underset{\sim}{x}\}=\gamma
$$

Let the observations $\underset{\sim}{x}$ come from a member of a family of sampling distributions indexed by a single parameter $\theta$, and assume that the corresponding $p^{\text {th }}$ quantile, $\xi_{p}$, is a monotonic function of $\theta$. If $\xi_{p}$ is decreasing, for example, then

$$
\operatorname{Pr}\left\{\xi_{p}(\theta) \leq U(\underset{\sim}{X}) \mid \underset{\sim}{x}=\underset{\sim}{x}\right\}=\operatorname{Pr}\left\{\theta \geq \xi_{p}^{-1}[U(\underset{\sim}{X})] \underset{\sim}{x}=\underset{\sim}{x}\right\},
$$

and this is equal to $\gamma$ if and only if $\xi_{p}{ }^{-1}[U(X)]$ is the $(1-\gamma)^{\text {th }}$ quantile of

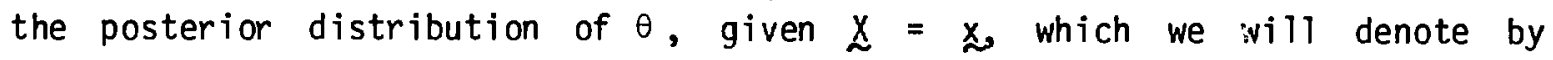
$\delta_{1-\gamma}\left(x_{\sim}\right)$. Thus, 


$$
U(\underline{x})=\xi_{p}\left(\delta_{1-\gamma}(\underline{x})\right)
$$

In the same way, we find

$$
L(x)=\xi_{1-p}\left(\delta_{Y}(x)\right)
$$

Similar results are obtained when $\xi_{p}$ is an increasing function of $\theta$.

For all of the one-parameter sampling distributions considered in Sec. I of the appendix, the conjugate prior distribution for the scale parameter $\theta$ (or for $\beta=\theta^{\alpha}$ in the Weibull case) is a gamma distribution, with probability density function

$$
I(\theta)=\frac{b^{a_{\theta} a-1} e^{-b \theta}}{\Gamma(a)}, \quad \theta>0
$$

This means that the posterior distribution, computed according to Bayes' rule as

$$
\pi(\theta \mid x)=\frac{\pi(\theta) L(x \mid \theta)}{\int_{0}^{\infty} \pi(\theta) L(x \mid \theta) d \theta}
$$

turns out to be another gama distribution. $(L(x \mid \theta)$ is the likelihood function of the observations $x$, given $\theta$ ). Verification of this fact is algebraically tedious but straightforward. In particular, suppose the prior distribution has parameters $a$ and $b$ as in Eq. (A.22). When the sampling distribution of $X$ is exponential (or Weibull with shape parameter $\alpha$ ), the parameters of the posterior distribution of $\theta($ or $\beta)$ are $a+r$ and $b+z$, where $r$ is the number of uncensored observations and

$$
z=\sum_{i=1}^{r} x_{(i)}+(n-r) x_{(r)}
$$


for

$$
\left.z=\sum_{i=1}^{r} x_{(i)}^{\alpha}+(n-r) x_{(r)}^{\alpha}\right)
$$

When the sampling distribution of $X$ is a gamma distribution with shape parameter $\alpha$ and $r=n$, the parameters of the posterior distribution of $\theta$ are $n \alpha+a$ and $b+z$, where $z$ is given by Eq. (A.23).

We shall continue to assume that $a$ and $\alpha$ are half integers, and use the fact that if $T$ has a gamma distribution with parameters $k / 2$ and $\tau$, then $2 \tau T$ has a chi-square distribution with $k$ degrees of freedom. The $\gamma^{\text {th }}$ quantile of the distribution of $T$ is given by

$$
\delta_{\gamma}=\frac{x_{k, \gamma}^{2}}{2 \tau}
$$

$\xi_{p}(\theta)$ has been given for all the relevant distributions in Sec. I of this appendix. Combining these results according to Eqs. (A.20) and (A.21) leads immediately to Bayesian tolerance limits for these models.

a. For an exponential sampling distribution, $k=2(a+r)$ and $\tau=b+z$ in Eq. (A.25), so from Eq. (A.5),

$$
\begin{aligned}
& L(\underset{\sim}{x})=-\frac{2(b+z) \ln (P)}{x_{2(a+r),}^{2}} \\
& U(\underset{\sim}{x})=-\frac{2(b+z) \ln (1-P)}{x_{2(a+r), 1-\gamma}^{2}} .
\end{aligned}
$$

b. For a gamma sampling distribution, with $k=2(n \alpha+a), \tau=b+z$, and using Eq. (A.10), ,

$$
L(\underset{\sim}{\sim})=\frac{(b+z) x_{2 \alpha, 1-p}^{2}}{x_{2(n \alpha+a), \gamma}^{2}}
$$




$$
U(\underset{\sim}{x})=\frac{(b+z) x_{2 \alpha, p}^{2}}{x_{2(n \alpha+a), 1-\gamma}^{2}}
$$

c. For a Weibull sampling distribution, with $z$ appropriateiy redefined [Eq. (A.24)], $k=2(a+r)$ and $\tau=b+z$ again, and

$$
\begin{aligned}
& L(\underset{\sim}{x})=\left(-\frac{2(b+z) \ln (P)}{x_{2(a+r), \gamma}^{2}}\right)^{1 / \alpha} \\
& U(\underset{\sim}{x})=\left(-\frac{2(b+z) \ln (1-P)}{x_{2(a+r), 1-\gamma}^{2}}\right)^{1 / \alpha} .
\end{aligned}
$$

Prior tolerance limits are available in each case by taking $n=r=z=0$.

IV. BAYESIAN LOGNORMAL MODEL

The conjugate prior for the two parameters of a normal distribution, $\mu$ and $\sigma$, is a three-parameter distribution called the normal-gamma distribution, $N \Gamma(x, m, q)$. Its probability density function is

$$
\pi(\mu, \sigma)=\sqrt{\frac{2 m}{\pi}\left(\frac{q}{m}\right)^{m-1}} \frac{\exp \left\{-\left[q+m(\mu-x)^{2} / 2 \sigma^{2}\right]\right\}}{\sigma^{m+1} \Gamma\left(\frac{m-1}{2}\right)} .
$$

The following transformation of variables is very useful:

$$
\begin{aligned}
& \tau=\frac{\sqrt{m}(\mu-x)}{\sigma} \\
& n=\frac{q}{\sigma^{2}}
\end{aligned}
$$

When the joint probability distribution of $\tau$ and $n$ is computed, it is found to factor into two familiar density functions, 


$$
\begin{aligned}
& f_{1}(\tau)=\frac{1}{\sqrt{2 \pi}} e^{-\tau^{2} / 2} \\
& f_{2}(n)=\frac{(1 / 2)_{n}^{S S-1} e^{-n / 2}}{\Gamma(S)},
\end{aligned}
$$

where $S=(m-1) / 2$. Thus, $\tau$ and $n$ are independent, $\tau$ has a standard normal distribution and $n$ has a chi square distribution with $2 S=m-1$ degrees of freedom.

Using Bayes' rule, it can be verified that if the prior distribution of $(\mu, \sigma)$ is taken to be a normal-gamma distribution with parameters $x, m$ and $q$, tne posterior distribution, given a sample $Y_{1}, \ldots Y_{n}$ from the $\left(\mu, \sigma^{2}\right)$ sampling distribution, is another normal-gamma distribution with parameters

$$
\begin{aligned}
& \bar{x}=\frac{m x+n \bar{y}}{m+n} \\
& \bar{m}=m+n \\
& \bar{q}=q+(n-1) s^{2}+\frac{m n}{m+n}(\bar{y}-x)^{2} .
\end{aligned}
$$

To compute a posterior upper tolerance limit we need $U(\underset{\sim}{(Y)}$ such that

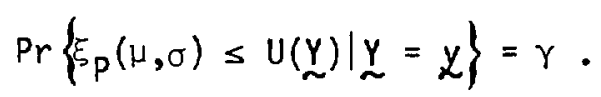

where $\xi_{p}(\mu, \sigma)$ is the $p^{\text {th }}$ quantile of the normal distribution with parameters $\mu$ and $\sigma$, thus $\xi_{p}(\mu, \sigma)=\mu+z_{p} \sigma$. The transformation (A.32) with the parameters of the posterior distribution $(\bar{x}, \bar{m}$ and $\bar{q})$ inverts to give us

$$
\begin{aligned}
& \mu=\bar{x}+\frac{\tau}{\sqrt{\bar{m}}} \sqrt{\frac{\bar{g}}{\eta}} \\
& \sigma=\sqrt{\frac{\bar{q}}{\eta}} .
\end{aligned}
$$


In terms of $\tau$ and $n$, then, we require $U(\underset{\sim}{)}$ ) such that

$$
\operatorname{Pr}\left\{\bar{x}+\sqrt{\frac{\overline{\bar{q}}}{\eta}}\left(\frac{\tau}{\sqrt{\mathrm{m}}}+z_{p}\right) \leq \underset{\sim}{U} \underset{\sim}{\underset{\sim}{Y}}=\underset{\sim}{y}\right\}=\gamma .
$$

Rearrangement leads to

$$
\operatorname{Pr}\left\{T_{\bar{m}-1}\left(\sqrt{\bar{m}} z_{p}\right) \leq \sqrt{\frac{\bar{m}(\bar{m}-1)}{\bar{q}}}(U(Y)-\bar{x})\right\}=\gamma,
$$

where

$$
T_{m-1}\left(\sqrt{m} z_{p}\right)=\frac{\tau+\sqrt{\bar{m}} z_{p}}{\sqrt{n /(\bar{m}-1)}}
$$

has a non-central $t$ distribution with $\bar{m}-1$ degrees of freedom and noncentrality parameter $\sqrt{\bar{m}} z_{p}$. Therefore, Eq. (A.34) implies that

$$
U(\underline{Y})=\bar{x}+\sqrt{\frac{\bar{q}}{\sqrt{m}-1}} \cdot \frac{t_{\bar{m}-1, \gamma\left(\sqrt{m} z_{p}\right)}}{\sqrt{\frac{\pi}{m}}},
$$

in which the last factor is recognizable as $K(\bar{m}, P, \gamma)$ from Eq. (A.17). Thus, the Bayesian tolerance limit looks much like the classical one of Eq. (A.18),

$$
U(\underline{x})=\exp \left\{\bar{X}+K(\bar{m}, P, Y) \sqrt{\frac{\bar{q}}{\bar{m}-1}}\right\},
$$

but $\bar{Y}$ has been replaced by a weighted average of $\bar{Y}$ and the prior "mean" $x, n$ by a sum $m+n$, and $S$ by $\sqrt{\bar{q} /(\bar{m}-1)}$, which also looks something like an average of a prior "variance" $s_{0}^{2}=q /(m-1)$ and the observed variance $s^{2}$, plus a term proportional to the square of the differences of the prior and observed means. (See Eqs. (A.33).)

The prior upper tolerance limit is similarly computed, starting with the definition 


$$
\operatorname{Pr}\left\{\xi_{p}(\mu, \sigma) \leq U\right\}=\gamma
$$

and using the prior $N \Gamma(x, m, q)$ distribution for $\mu$ and $\sigma$. The result is

$$
U=\exp \left\{x+K(m, P, Y) \sqrt{\frac{q}{m-1}}\right\} .
$$

\section{APPENDIX B}

\section{ACCURACY AND SAMPLE SIZE}

By definition, an upper $(P, Y)$ tolerance limit $U(X)$ has probability $Y$ of being an upper bound for $\xi_{p}$ or of bounding a fraction $P$ (or more) of the population from above. Although these two definitions are equivalent, the latter is a more direct interpretation of what we usually want when we compute tolerance limits. It is possible (especially when $P$ is large) for $U(X)$ to have large variance and to exceed $\xi_{p}$ by a large amount, yet $F(U(X))$ may still not be much bigger than $P$. Therefore,

$$
T(\underset{\sim}{X})=F(U(\underset{\sim}{X}))-P
$$

seems like a reasonable measure of the accuracy of $U(\underset{\sim}{X})$. In particular, knowing that, by definition, $\operatorname{Pr}[T(\underset{\sim}{X}) \geq 0\}=\gamma$, we will ask how large a sample is required to guarantee that $\operatorname{Pr}\{(X) \geq \Delta P>0\}$ is small, say not greater than $1-Y$.

For example, consider a Weibull model with known shape parameter $\alpha$. An upper $(P, Y)$ tolerance limit is provided by the statistic

$$
U(\underset{\sim}{x})=\left(-\frac{2 r \ln (1-P)}{\hat{\theta}^{c} x_{2 r, 1-\gamma}^{2}}\right)^{1 / \alpha} .
$$

Fixing $\triangle P<1-P$, we have

$$
\operatorname{Pr}\{F(U(X)) \geq P+\Delta P\}=\operatorname{Pr}\{(\underset{\sim}{(X)} \geq \xi P+\Delta P\}
$$




$$
\begin{aligned}
& =\operatorname{Pr}\left\{\left(-\frac{2 r \ln (1-P)}{\hat{\theta}^{\alpha} x_{2 r, 1-\gamma}^{2}}\right)^{1 / \alpha} \geq\left(-\frac{\ln (1-P-\Delta P)}{\theta^{\alpha}}\right)^{1 / \alpha}\right\} \\
& =\operatorname{Pr}\left\{\frac{2 r \theta^{\alpha}}{\hat{\theta}^{\alpha}} \geq x_{2 r, 1-\gamma}^{2} \frac{\ln (1-P-\Delta P)}{\ln (1-P)}\right\},
\end{aligned}
$$

and this is less than or equal to $1-\gamma$ only if the right-hand side exceeds the $\gamma^{\text {th }}$ quantile of a chi-square with $2 r$ degrees of freedom (the distribution of $\left.2 r \theta^{\alpha} / \hat{\theta}^{\alpha}\right)$. That is,

$$
\operatorname{Pr}\{F(U \underset{\sim}{(X)}) \geq P+\Delta P\} \leq 1-\gamma
$$

if and only if

$$
\frac{x_{2 r, \gamma}^{2}}{x_{2 r, 1-\gamma}^{2}} \leq \frac{\ln (1-P-\Delta P)}{\ln (1-P)} .
$$

For a fixed $P, \Delta P$ and $\gamma$, the left-hand side of the inequality (B.1) is a decreasing function of $r$, and the inequality can be satisfied provided that $r$ is sufficiently large. It is an increasing function of $\gamma$, and the right-hand side is a decreasing function of $\Delta P$, so 1 arger samples are required for greater accuracy. The right-hand side is actually increasing in $P$ for large $P$, implying that it is easier to estimate large percentiles than smaller ones. This reflects our choice of a measure of accuracy. Although $\operatorname{Var}(U(X))$ is increasing as $(\ln (1-P))^{2 / \alpha}$ as $P+1$, the variance of $F(U(X))$ is approximately

$$
\frac{c^{2}}{r}\left[\ln (1-P) e^{c \ln (1-P)}\right]^{2} \text {, }
$$

and this becomes small as $P \rightarrow 1$.

Lower tolerance. limits are accurate within $\Delta P$ if 


$$
\frac{x_{2 r, \gamma}^{2}}{x_{2 r, 1-\gamma}^{2}}=\frac{\ln (P j}{\ln (P+\Delta P)} \text {. }
$$

For fixed $P$ and $\Delta P$, the right-hand side of Eq. (B.2) is larger than the right-hand side of Eq. (B.1), so smaller samples are needed for comparable accuracy of lower tolerance limits.

Similar arguments lead to similar inequalities for tolerance limits computed under a gamma model with known shape parameter $a$. In order to have $\operatorname{Pr}\{F(U(X)) \geq P+\Delta P\} \leq 1-\gamma$, we need

$$
\frac{x_{2 \alpha n, \gamma}^{2}}{x_{2 \alpha n, I-\gamma}^{2}} \leq \frac{x_{2 \alpha, p+\Delta p}^{2}}{x_{2 \alpha, p}^{2}}
$$

The solution $n$ to Eq. (B.3) is a decreasing function of $\alpha$. For $P=\gamma=0.95$ and $P=0.025$, the minimum sample sizes as a function of $\alpha$ are

$$
\begin{array}{ll}
n=302 & \text { for } \alpha=0.5, \\
n=251 & \text { for } \alpha=1.0, \\
n=210 & \text { for } \alpha=2.0, \\
n=180 & \text { for } \alpha=4.0,
\end{array}
$$

For lower tolerance limits, $\operatorname{Pr}\{1-F(L(X)) \geq P+\Delta P\} \leq 1-\gamma$ if

$$
\frac{x_{2 \alpha n, \gamma}^{2}}{x_{2 \alpha n, 1-\gamma}^{2}} \leq \frac{x_{2 \alpha, 1-P}^{2}}{x_{2 \alpha, 1-P-\Delta P}^{2}}
$$

This is an increasing function of $\alpha$. For $P=\gamma=0.95$ and $P=0.025$, the smallest $n$ satisfying Eq. (B.4) goes from 13 for $\alpha=0.5$ to 54 for $\alpha=4.0$.

For the lognormal distribution we had

$$
U(\underset{\sim}{X})=\exp (\bar{Y}+K(n, P, Y) S)
$$


We now wish to choose $n$ so that

$$
\begin{aligned}
1-\gamma & \geq \operatorname{Pr}\{F(\underset{\sim}{(U)}) \geq P+\Delta P\} \\
& =\operatorname{Pr}\left\{\bar{Y}+K(n, P, \gamma) S z \mu+z_{P+\Delta P} \sigma\right\} \\
& =\operatorname{Pr}\left\{\sqrt{n} \frac{\bar{Y}-\mu}{\sigma}-\sqrt{n} z_{P+\Delta P} \geq-\sqrt{n} K(n, P, \gamma) \frac{S}{\sigma}\right\} \\
& =\operatorname{Pr}\left\{\frac{-\sqrt{n} \frac{\bar{Y}-\mu}{\sigma}+\sqrt{n} z_{P+\Delta P}}{\frac{S}{\sigma}} \leq \sqrt{n} K(n, P, \gamma)\right\} .
\end{aligned}
$$

The left-hand side of this last inequality again has a non-central $t$ distribution with $n-1$ degrees of freedom and non-centrality parameter $\sqrt{n} z_{p+p}$, so Eq. (B.5) implies that

$$
\begin{aligned}
\sqrt{n} K(n, P, \gamma) & \leq t_{n-1,1-\gamma}\left(\sqrt{n} z_{p+\Delta P}\right) \\
& =\sqrt{n} K(n, P+\Delta P, 1-\gamma) .
\end{aligned}
$$

It is difficult to compute the smallest value of $n$ satisfying Eq. (B.6) for fixed $P, \Delta P$ and $\gamma$, so these were not tabulated in Sec. VI.

In the Bayesian case we will require that

$$
\operatorname{Pr}\left\{\xi_{P+\Delta P} \leq U(\underset{\sim}{X}) \mid \underset{\sim}{X}=\underset{\sim}{x}\right\}
$$

or

$$
\operatorname{Pr}\left[\xi_{1-P}-\Delta P \geq L \underset{\sim}{(X)} \underset{\sim}{X}=\underset{\sim}{x}\right\}
$$

be small. For a Weibull sampling distribution with known shape parameter $\alpha$ and a gamma prior on the scale parameter $\theta$, we had 


$$
L \underset{\sim}{x})=\left(-\frac{2(b+z) \ln (P)}{x_{2(a+r), \gamma}^{2}}\right)^{1 / \alpha},
$$

where $z$ was defined by Eq. (A.24). With

$$
\xi_{1-P-\Delta P}=\left(-\frac{\ln (P+\Delta P)}{\theta^{\alpha}}\right)^{1 / \alpha},
$$

we get

$$
\begin{aligned}
\operatorname{Pr}\left\{\xi_{1-P-\Delta P} \geq L(x)\right\} & =\operatorname{Pr}\left\{-\frac{\ln (P+\Delta P)}{\theta^{\alpha}} \geq-\frac{2(b+z) \ln (P)}{x_{2(a+r), \gamma}^{2}}\right\} \\
& =\operatorname{Pr}\left\{2(b+z) \theta^{\alpha} \leq x_{2(a+r), \gamma}^{2} \frac{\ln (P+\Delta P)}{\ln (P)}\right\} .
\end{aligned}
$$

Since the posterior distribution of $\theta^{\alpha}$ is a gama distribution with parameters $a+r$ and $b+z$, the distribution of $2(b+z) \theta^{\alpha}$ is a chi-square with $2(a+r)$ degrees of freedom, so the above probability is less than $1-\gamma$ only if

$$
x_{2(a+r), \gamma}^{2} \frac{\ln (P+\Delta P)}{\ln (P)} \leq x_{2(a+r), 1-\gamma}^{2} .
$$

This leads to the requirement of Eq. (B.2) with $r$ replaced by a $+r$. Large values of the prior shape parameter a correspond to very narrow prior distributions (that is, to very precise prior knowledge). Usually a is small, and the required sample size is almost as 1 arge as for the classical case.

Working through the Bayesian versions of the other models gives very similar results. $r$ is replaced by $(a+r)$ in Eq. (B.1) for upper tolerance limits with a Weibull sampling distribution. $n$ is replaced by $n \alpha+a$ in Eqs. (B.3) and (B.4) for a gama sampling distribution, and $n$ is replaced by $m$ $+n$ in Eq. (B.6), where the normal-gamila prior had $m$ degrees of freedom. 
APPENDIX C

DATA SETS FOR EXAMPLES

I. DATA SET I

These data are failure-rate estimates for the failure of pumps to run normal mode, in failures per hour $(f / h)$. They are collected from several published sources.

$$
\text { Data: } \begin{aligned}
& 1.4 \times 10^{-7} \\
& 1.0 \times 10^{-6} \\
& 2.0 \times 10^{-6} \\
& 3.0 \times 10^{-6} \\
& 3.0 \times 10^{-6} \\
& 3.0 \times 10^{-6} \\
& 3.0 \times 10^{-6} \\
& 4.0 \times 10^{-6} \\
& 6.0 \times 10^{-6} \\
& 1.0 \times 10^{-5} \\
& 1.0 \times 10^{-5} \\
& 1.3 \times 10^{-5} \\
& 1.4 \times 10^{-4}
\end{aligned}
$$

Sample Size: 13

$$
\text { Mean: } 1.524 \times 10^{-5}
$$

Standard deviation: $3.769 \times 10^{-5}$

II. DATA SET II

These data are the first ten failure times, in hours, of 268 diaphragm values in the chemical and volume control systems of two nuclear reactor plants of the same design. The remaining values continued to operate normally for longer than 16833.4 hours. 


2827.6
3916.0
4416.2
6271.1
7211.9
8186.5
12203.7
13088.9
16144.6
16833.4

Uniensored sample size: 10

Total sample on test: 268 